\title{
On the Fukugita-Tanimoto-Yanagida Ansatz with Partially Non-degenerate Right-handed Majorana Neutrinos
}

\author{
Midori Obara * and Zhi-zhong Xing ${ }^{\dagger}$ \\ Institute of High Energy Physics, Chinese Academy of Sciences, \\ P.O. Box 918, Beijing 100049, China
}

\begin{abstract}
Taking three right-handed Majorana neutrino masses $M_{i}$ to be partially non-degenerate, we make a new analysis of the Fukugita-Tanimoto-Yanagida ansatz and confront it with current neutrino oscillation data. We determine the parameter space for cases (A) $M_{3}=M_{2} \neq M_{1}$, (B) $M_{2}=M_{1} \neq M_{3}$ and (C) $M_{1}=M_{3} \neq M_{2}$, and examine their respective deviations from the original $M_{1}=M_{2}=M_{3}$ case. The numerical constraints on three light neutrino masses, three neutrino mixing angles and three $\mathrm{CP}$-violating phases are also obtained, together with the predictions for the Jarlskog invariant of $\mathrm{CP}$ violation and the effective masses of the tritium beta decay and the neutrinoless double-beta decay.
\end{abstract}

11.30.Fs, 14.60.Lm, 14.60.Pq, 14.60.St

Typeset using REVTEX

*E-mail: midori@mail.ihep.ac.cn

${ }^{\dagger}$ E-mail: xingzz@mail.ihep.ac.cn 


\section{INTRODUCTION}

Very robust evidence for the existence of neutrino oscillations has recently been achieved from solar [1], atmospheric [2], reactor [3] and accelerator [4] neutrino experiments. Thanks to this exciting progress in neutrino physics, we are now convinced that neutrinos are massive and lepton flavors are mixed. The phenomenon of lepton flavor mixing can be described by a $3 \times 3$ unitary matrix $V$, the Maki-Nakagawa-Sakata (MNS) matrix [5], which contains three mixing angles $\left(\theta_{12}, \theta_{23}, \theta_{13}\right)$ and three $\mathrm{CP}$-violating phases $(\delta, \rho, \sigma)$. Four of these six parameters (i.e., $\theta_{12}, \theta_{23}, \theta_{13}$ and $\delta$ ), together with two neutrino mass-squared differences $\left(\Delta m_{21}^{2} \equiv m_{2}^{2}-m_{1}^{2}\right.$ and $\left.\Delta m_{32}^{2} \equiv m_{3}^{2}-m_{2}^{2}\right)$, can be extracted from the measurements of neutrino oscillations. A global analysis of current experimental data yields [6]

$$
\begin{array}{r}
0.25<\sin ^{2} \theta_{12}<0.38 \\
0.35<\sin ^{2} \theta_{23}<0.65 \\
\sin ^{2} \theta_{13}<0.03
\end{array}
$$

and

$$
\begin{aligned}
& 7.2 \times 10^{-5} \mathrm{eV}^{2} \leq \Delta m_{21}^{2} \leq 8.9 \times 10^{-5} \mathrm{eV}^{2} \\
& 2.1 \times 10^{-3} \mathrm{eV}^{2} \leq\left|\Delta m_{32}^{2}\right| \leq 3.1 \times 10^{-5} \mathrm{eV}^{2}
\end{aligned}
$$

at the $99 \%$ confidence level, but the Dirac CP-violating phase $\delta$ is entirely unrestricted at present. More accurate neutrino oscillation experiments are going to determine the size of $\theta_{13}$, the sign of $\Delta m_{32}^{2}$ and the magnitude of $\delta$. The proposed precision experiments for the tritium beta decay [7] and the neutrinoless double-beta decay [8] will help to probe the absolute mass scale of three light neutrinos and to constrain the Majorana CP-violating phases $\rho$ and $\sigma$.

Towards much better understanding of the neutrino mass spectrum and the neutrino mixing pattern indicated by Eqs. (1) and (2), many phenomenological ansätze of lepton mass matrices have recently been considered and discussed [9]. Among them, a particularly simple example [10] is to assume that both the charged-lepton mass matrix $M_{l}$ and the neutrino mass matrix $M_{\nu}$ are of the well-known Fritzsch texture [11] ${ }^{1}$. Incorporating the Fritzsch texture in the canonical seesaw mechanism [13] with three degenerate right-handed Majorana neutrinos, Fukugita, Tanimoto and Yanagida (FTY) [14] have proposed an interesting ansatz to account for current neutrino oscillation data. To be explicit, the FTY ansatz includes two assumptions: (1) both the charged-lepton mass matrix $M_{l}$ and the Dirac neutrino mass matrix $M_{\mathrm{D}}$ take the Fritzsch texture; and (2) the right-handed Majorana neutrino mass matrix $M_{\mathrm{R}}$ takes the form $M_{\mathrm{R}}=M_{0} 1$ with 1 being the $3 \times 3$ unity matrix (i.e., $M_{i}=M_{0}$ for $i=1,2$ and 3). Then the effective (left-handed) neutrino mass matrix $M_{\nu}$ in the FTY ansatz is given by

\footnotetext{
${ }^{1}$ See Ref. [12] for some earlier applications of the Fritzsch ansatz to the lepton sector. In these works, however, only the small-mixing-angle MSW solution to the solar neutrino problem was taken into account. This solution is now out of date.
} 


$$
M_{\nu}=M_{\mathrm{D}} M_{\mathrm{R}}^{-1} M_{\mathrm{D}}^{T}=\frac{M_{\mathrm{D}}^{2}}{M_{0}} .
$$

Unless a special assumption is further made [15], the texture of $M_{\nu}$ is no more of the Fritzsch form. Refs. [14] and [16] have shown that the FTY ansatz is compatible very well with current experimental data on solar and atmospheric neutrino oscillations.

The main purpose of this work is to go beyond the FTY ansatz by relaxing its second assumption; namely, the masses of three right-handed Majorana neutrinos are allowed to be partially non-degenerate. We consider three different cases: (A) $M_{3}=M_{2} \neq M_{1}$, (B) $M_{2}=M_{1} \neq M_{3}$, and (C) $M_{1}=M_{3} \neq M_{2}$. Indeed, the non-degeneracy or partial nondegeneracy of $M_{i}$ (for $i=1,2,3$ ) is a necessary condition to get the successful thermal leptogenesis [17] in order to account for the cosmological baryon-antibaryon asymmetry. See Ref. [18] for an explicit example which illustrates the correlation between the mass splitting of heavy right-handed Majorana neutrinos and the leptogenesis in the minimal seesaw model. Here we focus on the low-energy phenomenology of the generalized FTY ansatz - in particular, we are going to examine how the neutrino masses, flavor mixing angles and CP-violating phases are sensitive to the mass splitting parameters in cases (A), (B) and (C), which deviate from the original $M_{1}=M_{2}=M_{3}$ case in different ways. We shall discuss the possibilities to further extend the FTY ansatz to accommodate leptogenesis somewhere else.

The remaining part of our paper is organized as follows. In section II, we make some reasonable analytical approximations to calculate the neutrino mass matrix $M_{\nu}$, in which the mass splitting of any two heavy Majorana neutrinos has been taken into account. The neutrino mass spectrum and the MNS matrix can then be derived for each of the three cases. Section III is devoted to a detailed numerical analysis of the allowed parameter space in each case, and to the determination of three neutrino masses, three mixing angles and three CP-violating phases. We also obtain the predictions for the Jarlskog invariant of CP violation and the effective masses of the tritium beta decay and the neutrinoless double-beta decay. Finally, a brief summary of our main results is given in section IV.

\section{ANALYTICAL CALCULATIONS}

Let us assume that the charged-lepton mass matrix $M_{l}$ and the Dirac neutrino mass matrix $M_{\mathrm{D}}$ are both symmetric and of the Fritzsch texture:

$$
M_{l(\mathrm{D})}=\left(\begin{array}{ccc}
\mathbf{0} & C_{l(\mathrm{D})} & \mathbf{0} \\
C_{l(\mathrm{D})} & \mathbf{0} & B_{l(\mathrm{D})} \\
\mathbf{0} & B_{l(\mathrm{D})} & A_{l(\mathrm{D})}
\end{array}\right),
$$

in which only $A_{l(\mathrm{D})}$ is real and positive. $M_{l(\mathrm{D})}$ can be decomposed as $M_{l(\mathrm{D})}=P_{l(\mathrm{D})} \bar{M}_{l(\mathrm{D})} P_{l(\mathrm{D})}^{T}$, where $P_{l(\mathrm{D})}=\operatorname{Diag}\left\{e^{i\left[\varphi_{l(\mathrm{D})}-\phi_{l(\mathrm{D})}\right]}, e^{i \phi_{l(\mathrm{D})}}, 1\right\}$ with $\phi_{l(\mathrm{D})} \equiv \arg \left[B_{l(\mathrm{D})}\right]$ and $\varphi_{l(\mathrm{D})} \equiv \arg \left[C_{l(\mathrm{D})}\right]$, and

$$
\bar{M}_{l(\mathrm{D})}=\left(\begin{array}{ccc}
\mathbf{0} & \left|C_{l(\mathrm{D})}\right| & \mathbf{0} \\
\left|C_{l(\mathrm{D})}\right| & \mathbf{0} & \left|B_{l(\mathrm{D})}\right| \\
\mathbf{0} & \left|B_{l(\mathrm{D})}\right| & A_{l(\mathrm{D})}
\end{array}\right) .
$$


We diagonalize $\bar{M}_{l(D)}$ by using the orthogonal transformation $O_{l}^{\dagger} \bar{M}_{l} O_{l}^{*}=\operatorname{Diag}\left\{m_{e}, m_{\mu}, m_{\tau}\right\}$ or $O_{\mathrm{D}}^{\dagger} \bar{M}_{\mathrm{D}} O_{\mathrm{D}}^{*}=\operatorname{Diag}\left\{d_{1}, d_{2}, d_{3}\right\}$, where $m_{\alpha}$ (for $\alpha=e, \mu, \tau$ ) and $d_{i}$ (for $i=1,2,3$ ) denote the masses of charged leptons and the eigenvalues of $M_{\mathrm{D}}$, respectively. The elements of $O_{l}$ can be given in terms of two parameters $x_{l} \equiv m_{e} / m_{\mu}$ and $y_{l} \equiv m_{\mu} / m_{\tau}$, and the elements of $O_{\mathrm{D}}$ can similarly be given in terms of two parameters $x \equiv d_{1} / d_{2}$ and $y \equiv d_{2} / d_{3}$. Omitting the subscripts of $O_{l(\mathrm{D})}$ and those of $\left(x_{l}, y_{l}\right)$, we have [10]

$$
\begin{aligned}
& O_{11}=+\left[\frac{1-y}{(1+x)(1-x y)(1-y+x y)}\right]^{1 / 2}, \\
& O_{12}=-i\left[\frac{x(1+x y)}{(1+x)(1+y)(1-y+x y)}\right]^{1 / 2}, \\
& O_{13}=+\left[\frac{x y^{3}(1-x)}{(1-x y)(1+y)(1-y+x y)}\right]^{1 / 2}, \\
& O_{21}=+\left[\frac{x(1-y)}{(1+x)(1-x y)}\right]^{1 / 2}, \\
& O_{22}=+i\left[\frac{1+x y}{(1+x)(1+y)}\right]^{1 / 2}, \\
& O_{23}=+\left[\frac{y(1-x)}{(1-x y)(1+y)}\right]^{1 / 2}, \\
& O_{31}=-\left[\frac{x y(1-x)(1+x y)}{(1+x)(1-x y)(1-y+x y)}\right]^{1 / 2}, \\
& O_{32}=-i\left[\frac{y(1-x)(1-y)}{(1+x)(1+y)(1-y+x y)}\right]^{1 / 2}, \\
& O_{33}=+\left[\frac{(1-y)(1+x y)}{(1-x y)(1+y)(1-y+x y)}\right]^{1 / 2} .
\end{aligned}
$$

Note that $O_{12}, O_{22}$ and $O_{32}$ are imaginary, because the determinant of $\bar{M}_{l(\mathrm{D})}$ is negative. The mass matrix $M_{l(\mathrm{D})}$ is therefore diagonalized by the unitary matrix $V_{l(\mathrm{D})}=P_{l(D)} O_{l(\mathrm{D})}$. In the FTY ansatz, $M_{\mathrm{R}}=M_{0} \mathbf{1}$ is taken and $M_{\mathrm{D}}$ is assumed to be real (i.e., $P_{\mathrm{D}}=\mathbf{1}$ or $\phi_{\mathrm{D}}=\varphi_{\mathrm{D}}=0$, or equivalently $M_{\mathrm{D}}=\bar{M}_{\mathrm{D}}$ ). Such an assumption implies that one may diagonalize $M_{\nu}$ in Eq. (3) just by using the orthogonal transformation $V_{\nu}=O_{\mathrm{D}} Q$ with $Q=\operatorname{Diag}\{1, i, 1\}$ :

$$
V_{\nu}^{\dagger} M_{\nu} V_{\nu}^{*}=\frac{\left[Q^{\dagger}\left(O_{\mathrm{D}}^{\dagger} \bar{M}_{\mathrm{D}} O_{\mathrm{D}}^{*}\right) Q^{*}\right]^{2}}{M_{0}}=\frac{1}{M_{0}}\left(\begin{array}{ccc}
d_{1}^{2} & 0 & 0 \\
0 & d_{2}^{2} & 0 \\
0 & 0 & d_{3}^{2}
\end{array}\right),
$$

from which the neutrino masses $m_{i}=d_{i}^{2} / M_{0}$ can be obtained. The MNS matrix $V \equiv V_{l}^{\dagger} V_{\nu}$ turns out to be $V \equiv O_{l}^{\dagger} P_{l}^{\dagger} O_{\mathrm{D}} Q$.

Next we go beyond the FTY ansatz by allowing the masses of three heavy right-handed Majorana neutrinos (i.e., $M_{1}, M_{2}$ and $M_{3}$ ) to be partially non-degenerate. For simplicity, 
we keep the assumption of $M_{\mathrm{D}}=\bar{M}_{\mathrm{D}}$ (i.e., $M_{\mathrm{D}}$ is real and $P_{\mathrm{D}}=\mathbf{1}$ holds) and consider three different patterns of $M_{i}$ : (A) $M_{3}=M_{2} \neq M_{1}$, (B) $M_{2}=M_{1} \neq M_{3}$ and (C) $M_{1}=M_{3} \neq M_{2}$. We also assume $M_{\mathrm{R}}$ to be diagonal, real and positive in the flavor basis chosen above. Given $V_{\mathrm{D}}^{\dagger} M_{\mathrm{D}} V_{\mathrm{D}}^{*}=\operatorname{Diag}\left\{d_{1}, d_{2}, d_{3}\right\}$ with $V_{\mathrm{D}}=P_{\mathrm{D}} O_{\mathrm{D}}=O_{\mathrm{D}}$ as we have already discussed, the effective neutrino mass matrix $M_{\nu}$ can now be expressed as

$$
M_{\nu}=M_{\mathrm{D}} M_{\mathrm{R}}^{-1} M_{\mathrm{D}}^{T}=\bar{M}_{\mathrm{D}} M_{\mathrm{R}}^{-1} \bar{M}_{\mathrm{D}}^{T}=O_{\mathrm{D}} Q M_{\nu}^{\prime} Q^{T} O_{\mathrm{D}}^{T}
$$

where $Q=\operatorname{Diag}\{1, i, 1\}$ and

$$
M_{\nu}^{\prime} \equiv Q^{\dagger}\left(\begin{array}{ccc}
d_{1} & 0 & 0 \\
0 & d_{2} & 0 \\
0 & 0 & d_{3}
\end{array}\right) O_{\mathrm{D}}^{T} M_{\mathrm{R}}^{-1} O_{\mathrm{D}}\left(\begin{array}{ccc}
d_{1} & 0 & 0 \\
0 & d_{2} & 0 \\
0 & 0 & d_{3}
\end{array}\right) Q^{*}
$$

It is easy to check that Eq. (7) can simply be reproduced from Eqs. (8) and (9) by taking $M_{\mathrm{R}}=M_{0} \mathbf{1}$. If the masses of two right-handed Majorana neutrinos are not exactly degenerate, their small difference will enter the expression of $M_{\nu}^{\prime}$. To be more transparent, we specify cases (A), (B) and (C) as

$$
\begin{array}{rlrl} 
& & & \\
\text { Case (A) : } & & M_{\mathrm{R}}=\left(\begin{array}{ccc}
M_{1} & 0 & 0 \\
0 & M_{2} & 0 \\
0 & 0 & M_{2}
\end{array}\right), \\
\text { Case (B) : } & M_{\mathrm{R}}=\left(\begin{array}{ccc}
M_{1} & 0 & 0 \\
0 & M_{1} & 0 \\
0 & 0 & M_{2}
\end{array}\right), \\
\text { Case (C) : } & M_{\mathrm{R}}=\left(\begin{array}{ccc}
M_{1} & 0 & 0 \\
0 & M_{2} & 0 \\
0 & 0 & M_{1}
\end{array}\right),
\end{array}
$$

and then define a single mass splitting parameter $\delta_{12} \equiv\left(M_{2}-M_{1}\right) / M_{2}$ for each case. We obtain

$$
M_{\nu}^{\prime}=\frac{d_{3}^{2}}{M_{1}}\left(\begin{array}{ccc}
x^{2} y^{2} & 0 & 0 \\
0 & y^{2} & 0 \\
0 & 0 & 1
\end{array}\right)-\delta_{12} M_{\nu}^{\prime \prime}
$$

to the leading order of $\delta_{12}$, and list the explicit expressions of $M_{\nu}^{\prime \prime}$ for three different cases in TABLE I. $M_{\nu}^{\prime}$ can be diagonalized by the real orthogonal transformation $O_{\nu}^{\prime T} M_{\nu}^{\prime} O_{\nu}^{\prime}=\operatorname{Diag}\left\{m_{1}, m_{2}, m_{3}\right\}$, where $m_{i}$ (for $i=1,2,3$ ) denote the neutrino masses and $O_{\nu}^{\prime}=R_{23}\left(\theta_{23}^{\prime}\right) R_{12}\left(\theta_{12}^{\prime}\right) R_{13}\left(\theta_{13}^{\prime}\right)$ is the product of three canonical Euler rotation matrices. Thus it is the unitary matrix $V_{\nu}=O_{\mathrm{D}} Q O_{\nu}^{\prime}$ that diagonalizes the neutrino mass matrix $M_{\nu}$. The mass eigenvalues of $M_{\nu}^{\prime}$ and the rotation angles of $O_{\nu}^{\prime}$ are given in TABLE II, in which the sub-leading terms of $\mathcal{O}\left(x^{3}\right), \mathcal{O}\left(y^{3}\right), \mathcal{O}\left(x^{2} y\right), \mathcal{O}\left(x y^{2}\right)$ and $\mathcal{O}\left(\delta_{12}^{2}\right)$ have been neglected by assuming $0<x<1$ and $0<y<1$ (i.e., $d_{1}<d_{2}<d_{3}$ ) as well as $\left|\delta_{12}\right| \ll 1$. One may use the experimental values of $\Delta m_{21}^{2}$ and $\left|\Delta m_{32}^{2}\right|$ to constrain the parameter space of $x$ and $y$. Defining the ratio 


$$
R_{\nu} \equiv \frac{\Delta m_{21}^{2}}{\left|\Delta m_{32}^{2}\right|}=y_{\nu}^{2} \frac{1-x_{\nu}^{2}}{\left|1-y_{\nu}^{2}\right|}
$$

where $x_{\nu} \equiv m_{1} / m_{2}$ and $y_{\nu} \equiv m_{2} / m_{3}$, we can numerically verify that only $y_{\nu}<1$ is allowed as a consequence of $x<1$ and $y<1$.

Taking into account the mass splitting effect, we now express the MNS matrix $V=V_{l}^{\dagger} V_{\nu}$ as $V=O_{l}^{\dagger} P_{l}^{\dagger} O_{\mathrm{D}} Q O_{\nu}^{\prime}$, where $P_{l}$ is taken to be $P_{l}=\operatorname{Diag}\left\{e^{i \alpha}, e^{i \beta}, 1\right\}$ with $\alpha=\phi_{l}-\varphi_{l}$ and $\beta=-\phi_{l}$. $V$ totally contains seven parameters: $x_{l}, y_{l}, x, y, \delta_{12}, \alpha$ and $\beta$, among which $x_{l} \approx 0.00484$ and $y_{l} \approx 0.0595$ [19] are already known to a high degree of accuracy. Given the value of $\delta_{12}$, one is able to constrain the ranges of $(x, y)$ and $(\alpha, \beta)$ by using current experimental data on $R_{\nu}$ and $\left(\theta_{12}, \theta_{23}, \theta_{13}\right)$. To be specific, here we make use of the following parametrization of $V[20]$ :

$$
V=\left(\begin{array}{ccc}
c_{12} c_{13} & s_{12} c_{13} & s_{13} \\
-c_{12} s_{23} s_{13}-s_{12} c_{23} e^{-i \delta} & -s_{12} s_{23} s_{13}+c_{12} c_{23} e^{-i \delta} & s_{23} c_{13} \\
-c_{12} c_{23} s_{13}+s_{12} s_{23} e^{-i \delta} & -s_{12} c_{23} s_{13}-c_{12} s_{23} e^{-i \delta} & c_{23} c_{13}
\end{array}\right)\left(\begin{array}{ccc}
e^{i \rho} & 0 & 0 \\
0 & e^{i \sigma} & 0 \\
0 & 0 & 1
\end{array}\right)
$$

where $c_{i j} \equiv \cos \theta_{i j}, s_{i j} \equiv \sin \theta_{i j}$ (for $i j=12,23,13$ ), $\delta$ denotes the Dirac CP-violating phase, $\rho$ and $\sigma$ stand for the Majorana CP-violating phases. Then

$$
\sin ^{2} \theta_{12}=\frac{\left|V_{e 2}\right|^{2}}{1-\left|V_{e 3}\right|^{2}}, \quad \sin ^{2} \theta_{23}=\frac{\left|V_{\mu 3}\right|^{2}}{1-\left|V_{e 3}\right|^{2}}, \quad \sin ^{2} \theta_{13}=\left|V_{e 3}\right|^{2} .
$$

The leptonic Jarlskog invariant of CP violation [21] reads $\mathcal{J}=s_{12} c_{12} s_{23} c_{23} s_{13} c_{13}^{2} \sin \delta$, which only depends on the Dirac phase $\delta$. On the other hand, the effective masses of the tritium beta decay $\langle m\rangle_{e}$ and the neutrinoless double-beta decay $\langle m\rangle_{e e}$ are given by

$$
\langle m\rangle_{e}^{2}=\sum_{i=1}^{3}\left(m_{i}^{2}\left|V_{e i}\right|^{2}\right)=m_{3}^{2}\left(x_{\nu}^{2} y_{\nu}^{2} c_{12}^{2} c_{13}^{2}+y_{\nu}^{2} s_{12}^{2} c_{13}^{2}+s_{13}^{2}\right)
$$

and

$$
\langle m\rangle_{e e}=\left|\sum_{i=1}^{3}\left(m_{i} V_{e i}^{2}\right)\right|=m_{3}\left|x_{\nu} y_{\nu} c_{12}^{2} c_{13}^{2} e^{2 i \rho}+y_{\nu} s_{12}^{2} c_{13}^{2} e^{2 i \sigma}+s_{13}^{2}\right|
$$

One can see that the Dirac phase $\delta$ does not appear in $\langle m\rangle_{e e}$, as guaranteed by the phase convention of $V$ in Eq. (13). The present experimental upper bounds on $\langle m\rangle_{e}$ and $\langle m\rangle_{e e}$ are $\langle m\rangle_{e}<2.1 \mathrm{eV}$ and $\langle m\rangle_{e e}<0.39 \mathrm{eV}$, respectively, at the $99 \%$ confidence level [6]. The future KATRIN experiment is expected to reach the sensitivity $\langle m\rangle_{e} \simeq 0.2 \mathrm{eV}[7]$. On the other hand, the new experiments towards searching for the neutrinoless double-beta decay may reach the sensitivity $\langle m\rangle_{e e} \sim \mathcal{O}\left(10^{-2}\right) \mathrm{eV}[8]$.

\section{NUMERICAL ANALYSIS}

We proceed to do a numerical analysis of the generalized FTY ansatz with non-vanishing $\delta_{12}$. The neutrino mass spectrum and the MNS matrix depend on seven free parameters: 
$d_{3}, x, y, \alpha, \beta, M_{1}$ and $\delta_{12}$. These parameters may more or less get constrained from the experimental data given in Eqs. (1) and (2). In the $\delta_{12}=0$ case, which corresponds to the original FTY ansatz, an updated numerical analysis yields $\omega \equiv d_{3}^{2} / M_{1}\left(=m_{3}\right) \approx$ $(47 \sim 56) \mathrm{meV}, x\left(=\sqrt{x_{\nu}}\right) \approx 0.22 \sim 0.56, y\left(=\sqrt{y_{\nu}}\right) \approx 0.39 \sim 0.45, \alpha \approx 0 \sim 2 \pi$ and $\beta \approx 0.61 \pi \sim 1.4 \pi$. These results allow us to make the following predictions:

$$
\begin{aligned}
& \sin ^{2} \theta_{23} \approx 0.35 \sim 0.49, \quad \sin ^{2} \theta_{12} \approx 0.25 \sim 0.38, \quad \sin ^{2} \theta_{13} \approx 0.0050 \sim 0.030 \\
& R_{\nu} \approx 0.023 \sim 0.041, \quad x_{\nu} \approx 0.049 \sim 0.32, \quad y_{\nu} \approx 0.15 \sim 0.20 \\
& \delta \approx-0.26 \pi \sim 0.26 \pi, \quad \rho \approx-0.12 \pi \sim 0.12 \pi, \quad \sigma \approx-0.16 \pi \sim 0.16 \pi \\
& \langle m\rangle_{e} \approx(5.8 \sim 11) \mathrm{meV}, \quad\langle m\rangle_{e e} \approx(2.8 \sim 6.6) \mathrm{meV}, \quad \mathcal{J} \approx(-2.3 \sim 2.3) \times 10^{-2}
\end{aligned}
$$

Note that the absolute scales of $d_{3}$ and $M_{1}$ cannot separately be determined from our analysis. FIG. 1 shows the allowed regions of $\sin ^{2} \theta_{12}$ vs $\sin ^{2} \theta_{23}, R_{\nu}$ vs $\sin ^{2} \theta_{13}$ and $\delta$ vs $\langle m\rangle_{e e}$ in the $\delta_{12}=0$ case. To get a ball-park feeling of the effect of non-vanishing $\delta_{12}$ on neutrino masses and lepton flavor mixing, we may carry out a general numerical calculation by allowing $\delta_{12}$ to vary between -1 and +1 . Then we obtain the generous constraints on $\delta_{12}$ and $\omega$ as follows:

$$
\begin{array}{ll}
\text { Case }(\mathrm{A}): & \delta_{12} \leq 0.41, \quad \omega \leq 8.7 \times 10^{-2} \mathrm{eV} ; \\
\text { Case }(\mathrm{B}): \quad-0.82 \leq \delta_{12} \leq 0.99, \quad 3.2 \times 10^{-2} \mathrm{eV} \leq \omega \leq 2.2 \times 10^{-1} \mathrm{eV} \\
\text { Case }(\mathrm{C}): \quad-0.81 \leq \delta_{12} \leq 0.52, \quad 4.1 \times 10^{-2} \mathrm{eV} \leq \omega \leq 6.3 \times 10^{-2} \mathrm{eV}
\end{array}
$$

We have found that the parameter space in the $\delta_{12} \neq 0$ case is essentially distinguishable from that in the $\delta_{12}=0$ case, provided $\left|\delta_{12}\right|$ is of $\mathcal{O}(0.1)$ or larger. However, it seems more interesting to consider a small mass splitting between two heavy right-handed Majorana neutrinos in model building; i.e., $\left|\delta_{12}\right| \sim \mathcal{O}(0.1)$. Thus we shall fix $\left|\delta_{12}\right|=0.25$ as a typical input in our subsequent numerical calculations. Such a choice of $\left|\delta_{12}\right|$ implies that the analytical approximations made in Eq. (11) and TABLE II are valid.

TABLE III shows the allowed ranges of model parameters in cases $(\mathrm{A}),(\mathrm{B})$ and $(\mathrm{C})$ with $\left|\delta_{12}\right|=0.25$. Note that cases $\left(\mathrm{A}^{ \pm}\right),\left(\mathrm{B}^{ \pm}\right)$or $\left(\mathrm{C}^{ \pm}\right)$correspond to the positive and negative values of $\delta_{12}$ in this table, where the predictions for a number of observables are also listed. Some comments are in order.

- The model parameters $\omega$ and $y$ are restricted to the relatively narrow ranges. The allowed region of $x$ is somehow larger than that of $y$ in all cases, implying that the flavor mixing angle $\theta_{12}$ is less constrained than $\theta_{23}$ in the generalized FTY ansatz. There is little limitation to the phase parameter $\alpha$, although $\alpha=\pi$ has been ruled out in cases $\left(\mathrm{A}^{ \pm}\right),\left(\mathrm{B}^{-}\right)$and $\left(\mathrm{C}^{-}\right)$. In comparison, the phase parameter $\beta$ always takes values around $\pi$ to guarantee $\sin ^{2} \theta_{23}>0.35$.

- The maximal atmospheric neutrino mixing (i.e., $\theta_{23}=\pi / 4$ or $\sin ^{2} \theta_{23}=0.5$ ) can only be achieved in cases $\left(\mathrm{B}^{+}\right)$and $\left(\mathrm{C}^{+}\right)$. In all cases, $\sin ^{2} \theta_{12}$ is not well restricted. But the smallest neutrino mixing angle $\theta_{13}$ has an lower bound in each case: $\theta_{13}>3.76^{\circ}$ $\left(\mathrm{A}^{+}\right), 3.85^{\circ}\left(\mathrm{A}^{-}\right), 2.43^{\circ}\left(\mathrm{B}^{+}\right), 5.13^{\circ}\left(\mathrm{B}^{-}\right), 2.81^{\circ}\left(\mathrm{C}^{+}\right)$and $4.66^{\circ}\left(\mathrm{C}^{-}\right)$. If a reactor neutrino oscillation experiment can reach the sensitivity $\sin ^{2} 2 \theta_{13} \sim 1 \%$ (or equivalently $\theta_{13} \sim 2.87^{\circ}$ ), it will be able to test our prediction for $\theta_{13}$. 
- The ranges of three $\mathrm{CP}$-violating phases are not very restrictive. In particular, the CP-conserving case (i.e., $\delta=\rho=\sigma=0$ ) cannot be ruled out at present. The allowed range of $\mathcal{J}$ is roughly the same in all six cases. It is in principle possible to detect $|\mathcal{J}| \sim \mathcal{O}\left(10^{-2}\right)$ in the future long-baseline neutrino oscillation experiments.

- Just as we have expected, the neutrino mass spectrum has a normal hierarchy with $m_{3} \approx \sqrt{\left|\Delta m_{32}^{2}\right|} \sim 0.05 \mathrm{eV}$. The magnitude of $\langle m\rangle_{e}$ is therefore strongly suppressed, at most of $\mathcal{O}\left(10^{-2}\right) \mathrm{eV}$, which cannot be probed by the proposed KATRIN experiment. In comparison, the magnitude of $\langle m\rangle_{e e}$ is at the level of a few $\mathrm{meV}$, which might be measured in the future neutrinoless double-beta decay experiments.

To see how the parameter space changes from the $\delta_{12}=0$ case to the $\delta_{12}= \pm 0.25$ cases, we plot the allowed regions of $\sin ^{2} \theta_{12}$ vs $\sin ^{2} \theta_{23}, R_{\nu}$ vs $\sin ^{2} \theta_{13}$ and $\delta$ vs $\langle m\rangle_{e e}$ in FIGs. 1-4. The points in the scatter plots for different parameters may more or less correlate, because they all depend on the model parameters $\delta_{12}, \omega, x, y, \alpha$ and $\beta$. One can easily see from TABLE II that the magnitude of $\theta_{23}^{\prime}$ is suppressed by the smallness of $x y$ in case $(\mathrm{A})$, hence the enhancement or suppression effect of $\delta_{12} \neq 0$ on $\theta_{23}$ is not obvious at all in FIG. 2. Similar arguments hold for $\theta_{12}$ in case (B) and $\theta_{13}$ in case (A). Note that the deviation of $m_{3}$ from $\omega$ in case $(\mathrm{C})$ is also suppressed by the smallness of $y$. These analytical features are consistent with the numerical results shown in FIGs. 2-4. We find that case (C) in FIG. 4 is most sensitive to the effect induced by $\delta_{12} \neq 0$.

It is worth mentioning that our results, similar to those obtained from the FTY ansatz, are essentially stable against radiative corrections from the seesaw scale to the electroweak scale or vice versa. The reason is simply that $m_{1}, m_{2}$ and $m_{3}$ have a clear normal hierarchy (or equivalently, $x_{\nu}<1$ and $y_{\nu}<1$ in TABLE III) [22]. Therefore, we have omitted the insignificant renormalization-group running effects on three light neutrino masses, three flavor mixing angles and three CP-violating phases in our calculations. Note that the structural hierarchy of $M_{\nu}$ is not strong, nor is that of $M_{\mathrm{D}}$. To see this point more clearly, we have evaluated the ratios of $A_{\mathrm{D}},\left|B_{\mathrm{D}}\right|$ and $\left|C_{\mathrm{D}}\right|$ to $d_{3}$ and listed them in TABLE III. Taking case $\left(\mathrm{B}^{+}\right)$for example, we have

$$
M_{\mathrm{D}} \sim d_{3}\left(\begin{array}{ccc}
\mathbf{0} & 0.21 \cdots 0.36 & \mathbf{0} \\
0.21 \cdots 0.36 & \mathbf{0} & 0.34 \cdots 0.55 \\
\mathbf{0} & 0.34 \cdots 0.55 & 0.68 \cdots 0.87
\end{array}\right)
$$

where the absolute value of $d_{3}$ is unrestricted (note that only $d_{3}^{2} / M_{1} \equiv \omega$ gets constrained from current experimental data, as shown in TABLE III). Comparing $M_{\mathrm{D}}$ with the Fritzschtype up-quark mass matrix [11]

$$
M_{\text {up }} \sim m_{t}\left(\begin{array}{ccc}
\mathbf{0} & 2.0 \times 10^{-4} & \mathbf{0} \\
2.0 \times 10^{-4} & \mathbf{0} & 6.5 \times 10^{-2} \\
\mathbf{0} & 6.5 \times 10^{-2} & 1
\end{array}\right)
$$

where $m_{u} / m_{c} \sim 0.0023$ and $m_{c} / m_{t} \sim 0.0042$ are typically input, one can observe that the hierarchy of $M_{\mathrm{D}}$ is much weaker than that of $M_{\text {up }}$. As $M_{l}$ is strongly hierarchical [10] and $M_{\mathrm{R}}$ is close to the unity matrix in our scenario, the weak hierarchy of $M_{\mathrm{D}}$ is the main source of large flavor mixing in the lepton sector. 
Finally, we stress that our work will make much more sense, if some tension or disagreement appears between the original FTY ansatz and more accurate neutrino oscillation data in the near future. One may also generalize the FTY ansatz by abandoning the exact mass degeneracy of three right-handed Majorana neutrinos and allowing for $M_{1} \sim M_{2} \sim M_{3}$. This case, which involves two small mass splitting parameters, can be regarded as a further extension of cases (A), (B) and (C) discussed in the present paper.

\section{SUMMARY}

We have generalized the interesting FTY ansatz by allowing the masses of three heavy right-handed Majorana neutrinos to be partially non-degenerate, and then investigated the phenomenological consequences of this mass splitting on the neutrino mass spectrum, flavor mixing angles and CP-violating phases. Three simple but typical cases have been considered in our analysis: (A) $M_{3}=M_{2} \neq M_{1}$, (B) $M_{2}=M_{1} \neq M_{3}$ and (C) $M_{1}=M_{3} \neq M_{2}$. The analytical approximations and numerical results show that there is the parameter space in every case and the mass splitting effect may play an important role in fitting the experimental data. We have also obtained the numerical predictions for the Jarlskog invariant of CP violation and the effective masses of the tritium beta decay and the neutrinoless doublebeta decay. Some of our results can be experimentally tested in the near future.

In our discussions, we have taken both the Dirac neutrino mass matrix $M_{\mathrm{D}}$ and the right-handed Majorana neutrino mass matrix $M_{\mathrm{R}}$ to be real. Hence there is no leptogenesis even though the mass degeneracy of three right-handed neutrinos is partially broken. It is of course interesting to introduce non-trivial complex phases into $M_{\mathrm{D}}$ and (or) $M_{\mathrm{R}}$, such that $\mathrm{CP}$ violation may appear in the lepton-number-violating and out-of-equilibrium decays

of heavy right-handed Majorana neutrinos. We shall elaborate this idea elsewhere, so as to accommodate baryogenesis via leptogenesis in a new extension of the FTY ansatz.

\section{ACKNOWLEDGMENTS}

We would like to thank S. Zhou for useful discussions. This work is supported in part by the National Natural Science Foundation of China. 


\section{REFERENCES}

[1] SNO Collaboration, Q.R. Ahmad et al., Phys. Rev. Lett. 87, 071301 (2001); Phys. Rev. Lett. 92, 181301 (2004).

[2] Super-Kamiokande Collaboration, Y. Fukuda et al., Phys. Rev. Lett. 81, 1562 (1998); Y. Ashie et al., Phys. Rev. Lett. 93, 101801 (2004).

[3] KamLAND Collaboration, K. Eguchi et al., Phys. Rev. Lett. 90, 021802 (2003); T. Araki et al., Phys. Rev. Lett. 94, 081801 (2005).

[4] K2K Collaboration, M.H. Ahn et al., Phys. Rev. Lett. 90, 041801 (2003); Phys. Rev. Lett. 94, 081802 (2005).

[5] Z. Maki, M. Nakagawa, and S. Sakata, Prog. Theor. Phys. 28, 870 (1962).

[6] A. Strumia and F. Vissani, hep-ph/0606054.

[7] KATRIN Collaboration, V.M. Lobashev et al., Nucl. Phys. A 719, 153 (2003).

[8] See, e.g., F.T. Avignone, Nucl. Phys. Proc. Suppl. 143, 233 (2005)

[9] For recent reviews with extensive references, see, e.g., H. Fritzsch and Z.Z. Xing, Prog. Part. Nucl. Phys. 45, 1 (2000); S.F. King, Rept. Prog. Phys. 67, 107 (2004); G. Altarelli and F. Feruglio, New J. Phys. 6, 106 (2004); Z.Z. Xing, Int. J. Mod. Phys. A 19, 1 (2004); R.N. Mohapatra et al., hep-ph/0510213, R.N. Mohapatra and A.Yu. Smirnov, hep-ph/0603118.

[10] Z.Z. Xing, Phys. Lett. B 550, 178 (2002); Z.Z. Xing and S. Zhou, Phys. Lett. B 593, 156 (2004).

[11] H. Fritzsch, Phys. Lett. B 73, 317 (1978); Nucl. Phys. B 155, 189 (1979).

[12] See, e.g., J.L. Hewett and T.G. Rizzo, Phys. Rev. D 33, 1519 (1986); A.J. Davies and X.G. He, Phys. Rev. D 46, 3208 (1992); M. Fukugita, M. Tanimoto, and T. Yanagida, Prog. Theor. Phys. 89, 263 (1993); K.S. Babu and Q. Shafi, Phys. Lett. B 311, 172 (1993); Y. Achiman and T. Greiner, Phys. Lett. B 329, 33 (1994).

[13] P. Minkowski, Phys. Lett. B 67, 421 (1977); T. Yanagida, in Proceedings of the Workshop on Unified Theory and the Baryon Number of the Universe, edited by O. Sawada and A. Sugamoto (KEK, Tsukuba, 1979), p. 95; M. Gell-Mann, P. Ramond, and R. Slansky, in Supergravity, edited by P. van Nieuwenhuizen and D. Freedman (North Holland, Amsterdam, 1979), p. 315; S.L. Glashow, in Quarks and Leptons, edited by M. Lévy et al. (Plenum, New York, 1980), p. 707; R.N. Mohapatra and G. Senjanovic, Phys. Rev. Lett. 44, 912 (1980).

[14] M. Fukugita, M. Tanimoto, and T. Yanagida, Phys. Lett. B 562, 273, (2003). Their original idea can be found in Ref. [12].

[15] Z.Z. Xing and S. Zhou, Phys. Lett. B 606, 145 (2005).

[16] S. Zhou and Z.Z. Xing, Eur. Phys. J. C 38, 495 (2005); M. Randhawa, G. Ahuja, and M. Gupta, hep-ph/0607074.

[17] M. Fukugita and T. Yanagida, Phys. Lett. B 174, 45 (1986).

[18] Z.Z. Xing and S. Zhou, hep-ph/0607302.

[19] S. Eidelman et al., Phys. Lett. B 592, 1 (2004).

[20] H. Fritzsch and Z.Z. Xing, Phys. Lett. B 517, 363 (2001).

[21] C. Jarlskog, Phys. Rev. Lett. 55, 1039 (1985).

[22] P.H. Chankowski and Z. Pluciennik, Phys. Lett. B 316, 312 (1993); K.S. Babu, C.N. Leung, and J. Pantaleone, Phys. Lett. B 319, 191 (1993); M. Tanimoto, Phys. Lett. B 
360, 41 (1995); N. Haba and N. Okamura, Eur. Phys. J. C 14, 347 (2000). For recent reviews, see: J.A. Casas, J.R. Espinosa, A. Ibarra, and I. Navarro, Nucl. Phys. B 573, 652 (2000); P.H. Chankowski and S. Pokorski, Int. J. Mod. Phys. A 17, 575 (2002); S. Antusch, J. Kersten, M. Lindner, M. Ratz, and M.A. Schmidt, JHEP 0503, 024 (2005); J.W. Mei, Phys. Rev. D 71, 073012 (2005). 


\section{TABLES}

TABLE I. The expressions of $M_{\nu}^{\prime \prime}$ for cases (A), (B) and (C). Here we have defined $\omega \equiv d_{3}^{2} / M_{1}$, $D_{\nu} \equiv(1+x)(1-x y)(1+y)(1-y+x y), D_{\nu}^{\prime} \equiv(1+x)(1-x y)(1+y), F_{x, y} \equiv \sqrt{x y\left(1-x^{2}\right)\left(1-y^{2}\right)}$, $F_{x, x y} \equiv \sqrt{y\left(1-x^{2}\right)\left(1-x^{2} y^{2}\right)}$ and $F_{y, x y} \equiv \sqrt{x\left(1-y^{2}\right)\left(1-x^{2} y^{2}\right)}$, where $x=d_{1} / d_{2}$ and $y=d_{2} / d_{3}$.

\begin{tabular}{|c|c|c|c|c|}
\hline Case & \multicolumn{3}{|c|}{$M_{\nu}^{\prime \prime}$} & Abbreviated functions \\
\hline (A) & & $\left(\begin{array}{ccc}x^{3} y^{2} F_{11}^{\mathrm{A}} & x y^{2} F_{y, x y} & -x y^{2} F_{x, y} \\
x y^{2} F_{y, x y} & y^{2} F_{22}^{\mathrm{A}} & x y^{2} F_{x, x y} \\
-x y^{2} F_{x, y} & x y^{2} F_{x, x y} & F_{33}^{\mathrm{A}}\end{array}\right)$ & & $\begin{array}{l}F_{11}^{\mathrm{A}} \equiv(1+y)\left(1-y-x^{2} y^{2}+y^{2}\right) \\
F_{22}^{\mathrm{A}} \equiv(1-x y)\left(1+x y+x^{2} y^{2}-y^{2}\right) \\
F_{33}^{\mathrm{A}} \equiv(1+x)\left(1-y^{2}+x y^{2}-x^{2} y^{2}\right)\end{array}$ \\
\hline (B) & $\frac{\omega}{D_{\nu}}$ & $\left(\begin{array}{cc}x^{3} y^{3} F_{11}^{\mathrm{B}} & x y^{3}(1-x) F_{y, x y} \\
x y^{3}(1-x) F_{y, x y} & y^{3} F_{22}^{\mathrm{B}} \\
-x y(1+x y) F_{x, y} & y(1-y) F_{x, x y}\end{array}\right.$ & $\left.\begin{array}{c}-x y(1+x y) F_{x, y} \\
y(1-y) F_{x, x y} \\
F_{33}^{\mathrm{B}}\end{array}\right)$ & $\begin{array}{l}F_{11}^{\mathrm{B}} \equiv(1-x)(1+x y)(1+y) \\
F_{22}^{\mathrm{B}} \equiv(1-x)(1-x y)(1-y) \\
F_{33}^{\mathrm{B}} \equiv(1+x)(1+x y)(1-y)\end{array}$ \\
\hline (C) & $\frac{\omega}{D_{\nu}^{\prime}}$ & $\left(\begin{array}{ccc}x^{3} y^{2} F_{11}^{\mathrm{C}} & x y^{2} F_{y, x y} & x y F_{x, y} \\
x y^{2} F_{y, x y} & y^{2} F_{22}^{\mathrm{C}} & y F_{x, x y} \\
x y F_{x, y} & y F_{x, x y} & F_{33}^{\mathrm{C}}\end{array}\right)$ & & $\begin{array}{l}F_{11}^{\mathrm{C}} \equiv\left(1-y^{2}\right) \\
F_{22}^{\mathrm{C}} \equiv\left(1-x^{2} y^{2}\right) \\
F_{33}^{\mathrm{C}} \equiv y\left(1-x^{2}\right)\end{array}$ \\
\hline
\end{tabular}

TABLE II. The mass eigenvalues and rotation angles of $M_{\nu}^{\prime}$ in cases (A), (B) and (C). Here the definitions of $\omega, F_{x, y}, F_{x, x y}$ and $F_{y, x y}$ are the same as those in TABLE I. We have neglected the sub-leading terms of $\mathcal{O}\left(x^{3}\right), \mathcal{O}\left(y^{3}\right), \mathcal{O}\left(x^{2} y\right), \mathcal{O}\left(x y^{2}\right)$ and $\mathcal{O}\left(\delta_{12}^{2}\right)$ in our analytical approximations.

\begin{tabular}{|l|l|l|}
\hline \hline Case & Mass eigenvalues of $M_{\nu}^{\prime}$ & Rotation angles of $M_{\nu}^{\prime}$ \\
\hline & $m_{1} \simeq \omega x^{2} y^{2}\left[1-\frac{x\left(1-y+y^{2}\right) \delta_{12}}{(1+x)(1-x y)(1-y+x y)}\right]$ & $\tan 2 \theta_{23}^{\prime} \simeq \frac{-2 x y^{2} F_{x, x y} \delta_{12}}{\left(1+x-2 y^{2}\right)-\left(1+x-2 y^{2}\right) \delta_{12}}$ \\
& $m_{2} \simeq \omega y^{2}\left[1-\frac{\left(1+x y-y^{2}\right) \delta_{12}}{(1+x)(1+y)(1-y+x y)}\right]$ & $\tan 2 \theta_{12}^{\prime} \simeq \frac{-2 x F_{y, x y} \delta_{12}}{\left(1+x-x^{2}-y^{2}\right)+\left(1-y^{2}\right) \delta_{12}}$ \\
& $m_{3} \simeq \omega\left[1-\frac{\left(1-y^{2}\right) \delta_{12}}{(1-x y)(1+y)(1-y+x y)}\right]$ & $\tan 2 \theta_{13}^{\prime} \simeq \frac{2 x y^{2} F_{x, y} \delta_{12}}{\left(1+x-y^{2}\right)-\left(1+x-y^{2}\right) \delta_{12}}$ \\
\hline & $m_{1} \simeq \omega x^{2} y^{2}\left[1-\frac{x y(1-x+x y) \delta_{12}}{(1+x)(1-x y)(1-y+x y)}\right]$ & $\tan 2 \theta_{23}^{\prime} \simeq \frac{-2 y(1-y) F_{x, x y} \delta_{12}}{\left(1+x-2 y^{2}\right)-(1+x-y) \delta_{12}}$ \\
& $m_{2} \simeq \omega y^{2}\left[1-\frac{y(1-x)(1-y) \delta_{12}}{(1+x)(1+y)(1-y+x y)}\right]$ & $\tan 2 \theta_{12}^{\prime} \simeq \frac{-2 x y(1-x) F_{y, x y} \delta_{12}}{\left(1+x-x^{2}-y^{2}\right)-y(1-x-y) \delta_{12}}$ \\
& $m_{3} \simeq \omega\left[1-\frac{(1-y+x y) \delta_{12}}{(1-x y)(1+y)(1-y+x y)}\right]$ & $\tan 2 \theta_{13}^{\prime} \simeq \frac{2 x y(1+x y) F_{x, y} \delta_{12}}{\left(1+x-y^{2}\right)-(1+x-y) \delta_{12}}$ \\
& $m_{1} \simeq \omega x^{2} y^{2}\left[1-\frac{x(1-y) \delta_{12}}{(1+x)(1-x y)}\right]$ & $\tan 2 \theta_{23}^{\prime} \simeq \frac{-2 y F_{x, x y} \delta_{12}}{\left(1+x+y-y^{2}\right)-y\left(1-y-x^{2}\right) \delta_{12}}$ \\
& $m_{2} \simeq \omega y^{2}\left[1-\frac{(1+x y) \delta_{12}}{(1+x)(1+y)}\right]$ & $\tan 2 \theta_{12}^{\prime} \simeq \frac{-2 x F_{y, x y} \delta_{12}}{\left(1+x+y-x^{2}\right)-\delta_{12}}$ \\
& $m_{3} \simeq \omega\left[1-\frac{y(1-x) \delta_{12}}{(1+y)(1-x y)}\right]$ & $\tan 2 \theta_{13}^{\prime} \simeq \frac{-2 x y F_{x, y} \delta_{12}}{(1+x+y)-y\left(1-x^{2}\right) \delta_{12}}$ \\
\hline \hline
\end{tabular}


TABLE III. The allowed ranges of free parameters and the predicted values of some observables in cases $(\mathrm{A}),(\mathrm{B})$ and $(\mathrm{C})$ with the typical input $\left|\delta_{12}\right|=0.25$. Here cases $\left(\mathrm{A}^{ \pm}\right),\left(\mathrm{B}^{ \pm}\right)$or $\left(\mathrm{C}^{ \pm}\right)$ correspond to the positive and negative values of $\delta_{12}$.

\begin{tabular}{|c|c|c|c|c|c|c|}
\hline \hline & $\left(\mathrm{A}^{+}\right)$ & $\left(\mathrm{A}^{-}\right)$ & $\left(\mathrm{B}^{+}\right)$ & $\left(\mathrm{B}^{-}\right)$ & $\left(\mathrm{C}^{+}\right)$ & $\left(\mathrm{C}^{-}\right)$ \\
\hline$\delta_{12}$ & 0.25 & -0.25 & 0.25 & -0.25 & 0.25 & -0.25 \\
$\omega(\mathrm{meV})$ & $62 \sim 75$ & $40 \sim 45$ & $57 \sim 72$ & $40 \sim 48$ & $49 \sim 60$ & $44 \sim 54$ \\
$x$ & $0.26 \sim 0.68$ & $0.20 \sim 0.47$ & $0.23 \sim 0.67$ & $0.21 \sim 0.51$ & $0.22 \sim 0.75$ & $0.21 \sim 0.45$ \\
$y$ & $0.37 \sim 0.43$ & $0.40 \sim 0.44$ & $0.36 \sim 0.41$ & $0.42 \sim 0.48$ & $0.42 \sim 0.51$ & $0.38 \sim 0.43$ \\
$\alpha(\pi)$ & $0 \sim 0.9$ & $0 \sim 0.9$ & $0 \sim 2.0$ & $0.013 \sim 0.70$ & $0 \sim 2.0$ & $0.019 \sim 0.85$ \\
$\beta(\pi)$ & $1.1 \sim 2.0$ & $1.1 \sim 2.0$ & & $1.3 \sim 2.0$ & $1.3 \sim 2.0$ \\
\hline \hline $\sin ^{2} \theta_{23}$ & $0.64 \sim 1.4$ & $0.63 \sim 1.4$ & $0.61 \sim 1.4$ & $0.67 \sim 1.4$ & $0.51 \sim 1.5$ & $0.69 \sim 1.3$ \\
$\sin ^{2} \theta_{12}$ & $0.25 \sim 0.34$ & $0.25 \sim 0.38$ & $0.25 \sim 0.38$ & $0.25 \sim 0.38$ & $0.25 \sim 0.36$ & $0.25 \sim 0.38$ \\
$\sin ^{2} \theta_{13}$ & $0.0043 \sim 0.030$ & $0.0045 \sim 0.030$ & $0.0018 \sim 0.030$ & $0.0080 \sim 0.030$ & $0.0024 \sim 0.030$ & $0.0066 \sim 0.030$ \\
$R_{\nu}$ & $0.023 \sim 0.042$ & $0.024 \sim 0.036$ & $0.024 \sim 0.042$ & $0.024 \sim 0.040$ & $0.024 \sim 0.042$ & $0.024 \sim 0.042$ \\
$m_{3}(\mathrm{meV})$ & $47 \sim 57$ & $50 \sim 56$ & $47 \sim 56$ & $47 \sim 56$ & $47 \sim 56$ & $47 \sim 56$ \\
$x_{\nu}$ & $0.076 \sim 0.45$ & $0.033 \sim 0.20$ & $0.052 \sim 0.43$ & $0.044 \sim 0.26$ & $0.058 \sim 0.56$ & $0.039 \sim 0.18$ \\
$y_{\nu}$ & $0.15 \sim 0.22$ & $0.15 \sim 0.19$ & $0.15 \sim 0.21$ & $0.15 \sim 0.20$ & $0.15 \sim 0.24$ & $0.15 \sim 0.20$ \\
$\delta(\pi)$ & $-0.26 \sim 0.25$ & $-0.26 \sim 0.25$ & $-0.30 \sim 0.30$ & $-0.23 \sim 0.23$ & $-0.29 \sim 0.29$ & $-0.24 \sim 0.23$ \\
$\rho(\pi)$ & $-0.13 \sim 0.13$ & $-0.12 \sim 0.12$ & $-0.18 \sim 0.18$ & $-0.10 \sim 0.10$ & $-0.16 \sim 0.16$ & $-0.09 \sim 0.09$ \\
$\sigma(\pi)$ & $-0.16 \sim 0.16$ & $-0.15 \sim 0.15$ & $-0.20 \sim 0.20$ & $-0.13 \sim 0.13$ & $-0.19 \sim 0.18$ & $-0.13 \sim 0.13$ \\
$\langle m\rangle_{e}(\mathrm{meV})$ & $5.5 \sim 10$ & $5.7 \sim 11$ & $4.9 \sim 11$ & $6.5 \sim 11$ & $5.2 \sim 11$ & $6.3 \sim 11$ \\
$\langle m\rangle_{e e}(\mathrm{meV})$ & $2.9 \sim 6.7$ & $2.7 \sim 5.8$ & $2.7 \sim 7.6$ & $2.9 \sim 6.1$ & $2.7 \sim 8.0$ & $2.9 \sim 5.5$ \\
$\mathcal{J}\left(10^{-2}\right)$ & $-2.1 \sim 2.2$ & $-2.1 \sim 2.3$ & $-2.1 \sim 2.1$ & $-2.3 \sim 2.2$ & $-2.2 \sim 2.1$ & $-2.1 \sim 2.1$ \\
\hline \hline$A_{\mathrm{D}} / d_{3}$ & $0.68 \sim 0.86$ & $0.65 \sim 0.78$ & $0.68 \sim 0.87$ & $0.64 \sim 0.78$ & $0.63 \sim 0.87$ & $0.68 \sim 0.78$ \\
$\mid B_{\mathrm{D}} / / d_{3}$ & $0.34 \sim 0.55$ & $0.45 \sim 0.58$ & $0.34 \sim 0.55$ & $0.43 \sim 0.57$ & $0.32 \sim 0.58$ & $0.45 \sim 0.55$ \\
$\mid C_{\mathrm{D}} / / d_{3}$ & $0.24 \sim 0.38$ & $0.22 \sim 0.33$ & $0.21 \sim 0.36$ & $0.24 \sim 0.36$ & $0.25 \sim 0.47$ & $0.21 \sim 0.32$ \\
\hline \hline
\end{tabular}




\section{FIGURES}
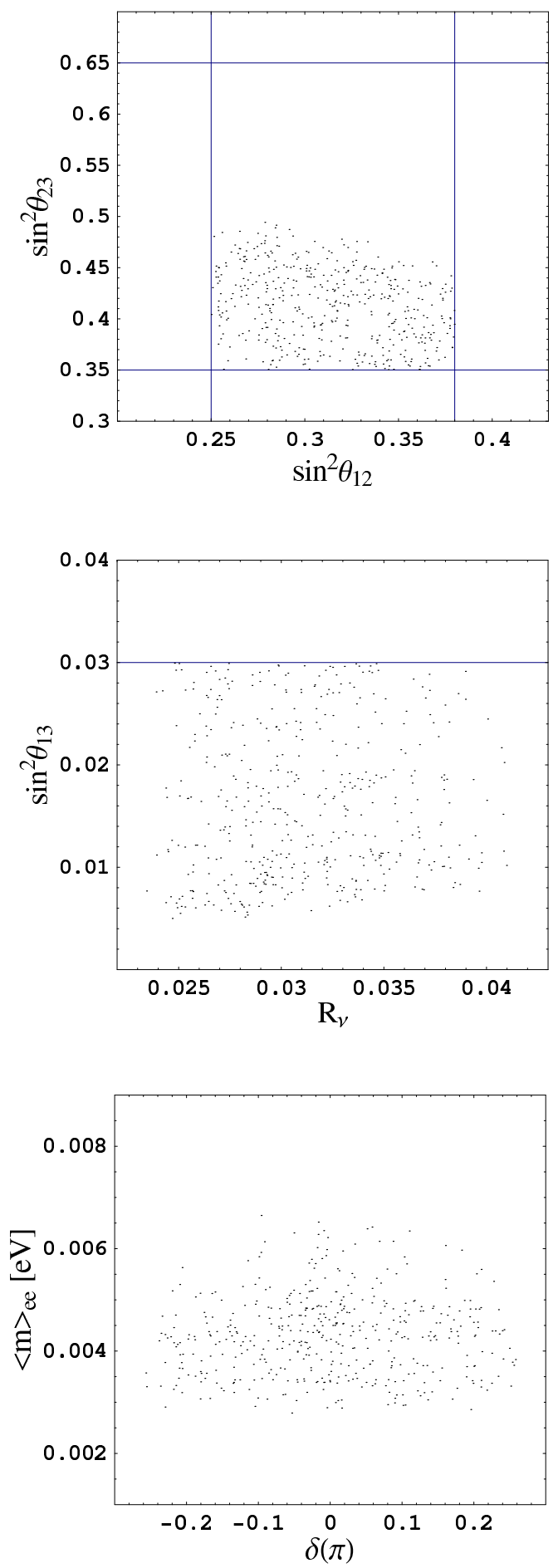

FIG. 1. The allowed regions of $\sin ^{2} \theta_{12}$ vs $\sin ^{2} \theta_{23}, R_{\nu}$ vs $\sin ^{2} \theta_{13}$ and $\delta$ vs $\langle m\rangle_{e e}$ in the $\delta_{12}=0$ (original FTY) case. 

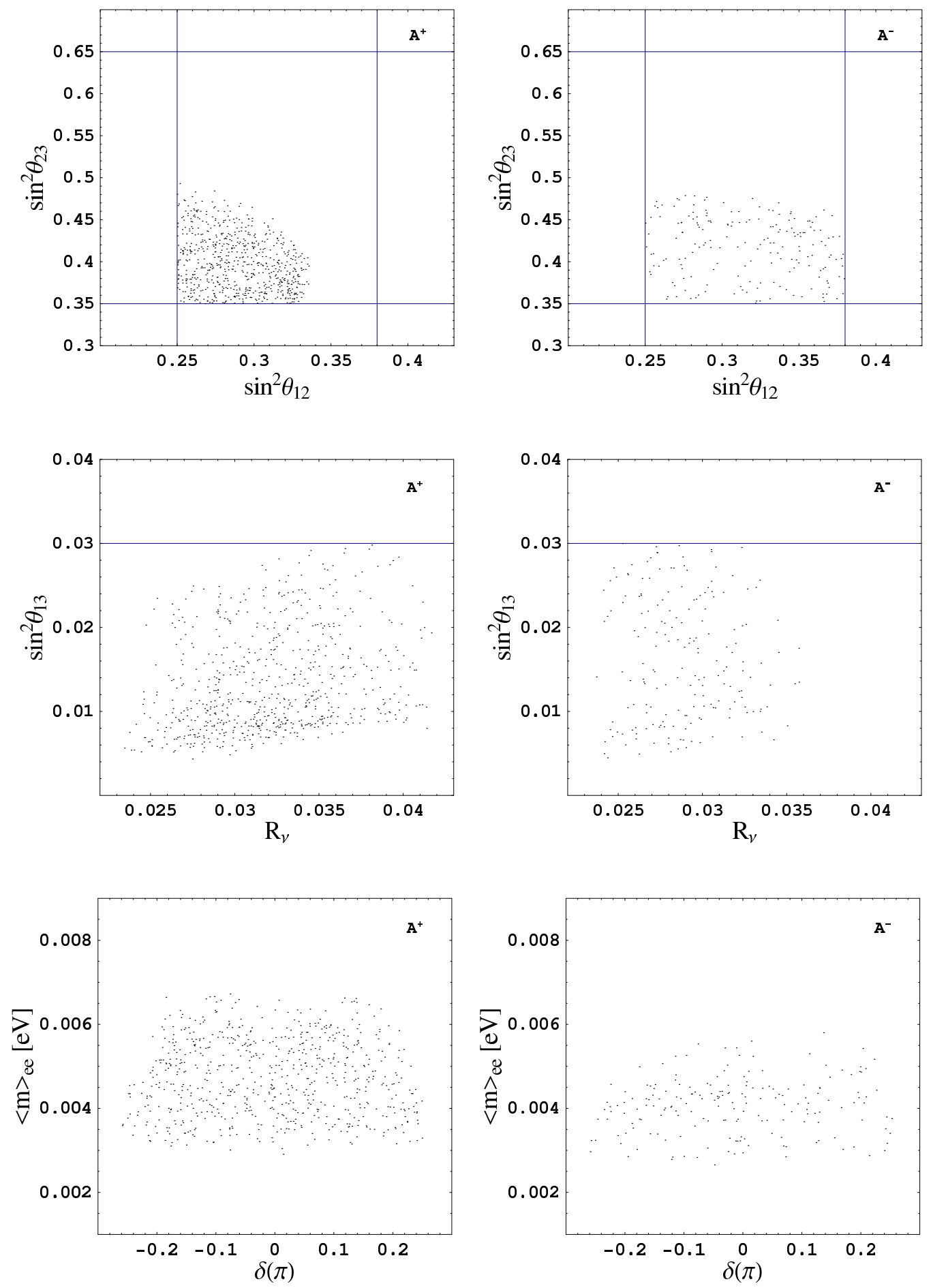

FIG. 2. The allowed regions of $\sin ^{2} \theta_{12}$ vs $\sin ^{2} \theta_{23}, R_{\nu}$ vs $\sin ^{2} \theta_{13}$ and $\delta$ vs $\langle m\rangle_{e e}$ in cases $\left(\mathrm{A}^{+}\right)$ and $\left(\mathrm{A}^{-}\right)$with $\left|\delta_{12}\right|=0.25$. 

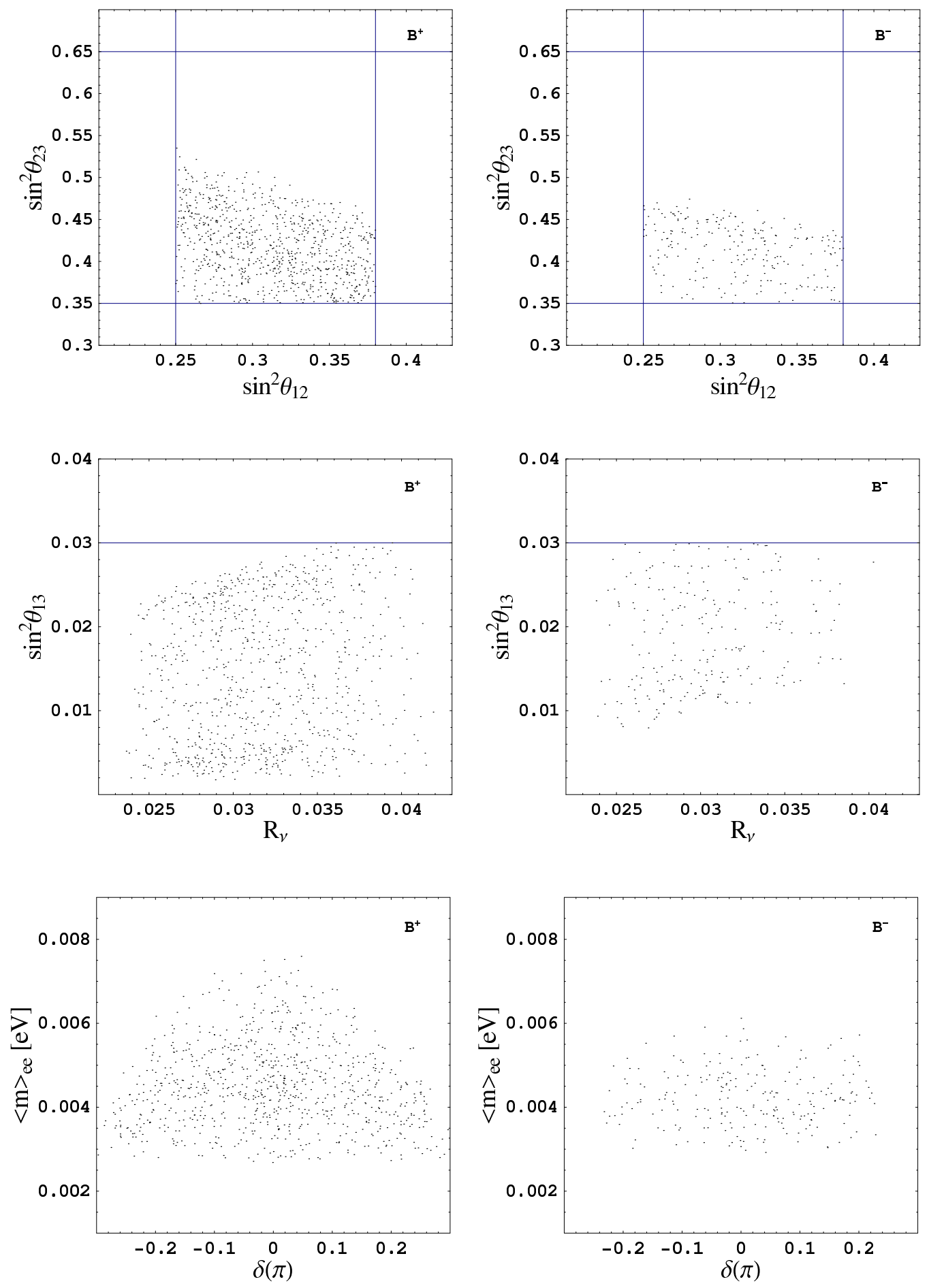

FIG. 3. The allowed regions of $\sin ^{2} \theta_{12}$ vs $\sin ^{2} \theta_{23}, R_{\nu}$ vs $\sin ^{2} \theta_{13}$ and $\delta$ vs $\langle m\rangle_{e e}$ in cases $\left(\mathrm{B}^{+}\right)$ and $\left(\mathrm{B}^{-}\right)$with $\left|\delta_{12}\right|=0.25$. 

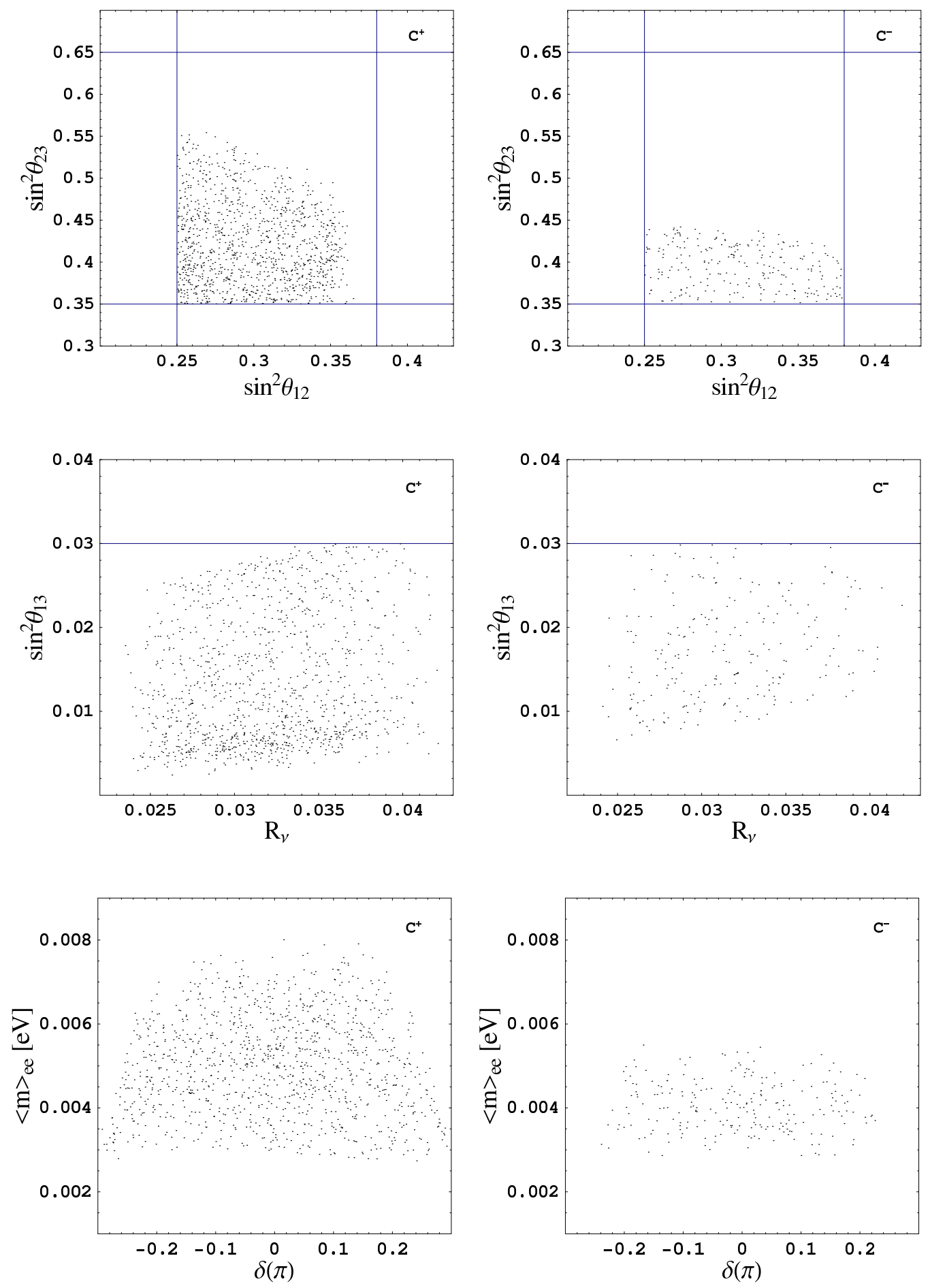

FIG. 4. The allowed regions of $\sin ^{2} \theta_{12}$ vs $\sin ^{2} \theta_{23}, R_{\nu}$ vs $\sin ^{2} \theta_{13}$ and $\delta$ vs $\langle m\rangle_{e e}$ in cases $\left(\mathrm{C}^{+}\right)$ and $\left(\mathrm{C}^{-}\right)$with $\left|\delta_{12}\right|=0.25$. 